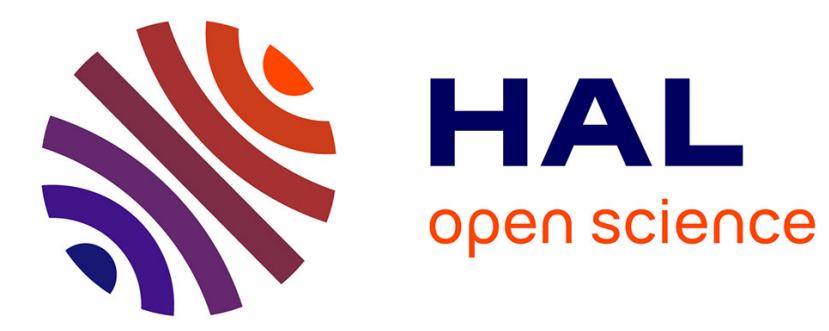

\title{
Multi-resonant silicon nanoantennas by evolutionary multi-objective optimization
}

Peter Wiecha, Arnaud Arbouet, Aurélie Lecestre, Guilhem Larrieu, Vincent Paillard

\section{- To cite this version:}

Peter Wiecha, Arnaud Arbouet, Aurélie Lecestre, Guilhem Larrieu, Vincent Paillard. Multi-resonant silicon nanoantennas by evolutionary multi-objective optimization. Computational Optics II, May 2018, Frankfurt, Germany. pp.1069402, 10.1117/12.2315123 . hal-02484255

\section{HAL Id: hal-02484255 \\ https://hal.science/hal-02484255}

Submitted on 19 Feb 2020

HAL is a multi-disciplinary open access archive for the deposit and dissemination of scientific research documents, whether they are published or not. The documents may come from teaching and research institutions in France or abroad, or from public or private research centers.
L'archive ouverte pluridisciplinaire HAL, est destinée au dépôt et à la diffusion de documents scientifiques de niveau recherche, publiés ou non, émanant des établissements d'enseignement et de recherche français ou étrangers, des laboratoires publics ou privés. 


\title{
Multi-resonant silicon nanoantennas by evolutionary multi-objective optimization
}

\author{
Peter R. Wiecha ${ }^{\mathrm{a}}$, Arnaud Arbouet ${ }^{\mathrm{a}}$, Christian Girard ${ }^{\mathrm{a}}$, Aurélie Lecestre ${ }^{\mathrm{b}}$, Guilhem Larrieu ${ }^{\mathrm{b}}$, \\ and Vincent Paillard ${ }^{\mathrm{a}}$
}

\author{
${ }^{a}$ CEMES, Université de Toulouse, CNRS, Toulouse, France \\ ${ }^{b}$ LAAS, Université de Toulouse, CNRS, INP, Toulouse, France
}

\begin{abstract}
Photonic nanostructures have attracted a tremendous amount of attention in the recent past. Via their size, shape and material it is possible to engineer their optical response to user-defined needs. Tailoring of the optical response is usually based on a reference geometry for which subsequent variations to the initial design are applied. Such approach, however, might fail if optimum nanostructures for complex optical responses are searched. As example we can mention the case of complex structures with several simultaneous optical resonances. We propose an approach to tackle the problem in the inverse way: In a first step we define the desired optical response as function of the nanostructure geometry. This response is numerically evaluated using the Green Dyadic Method for fully retarded electro-dynamical simulations. Eventually, we optimize multiple of such objective functions concurrently, using an evolutionary multi-objective optimization algorithm, which is coupled to the electro-dynamical simulations code. A great advantage of this optimization technique is, that it allows the implicit and automatic consideration of technological limitations like the electron beam lithography resolution. Explicitly, we optimize silicon nanostructures such that they provide two user-defined resonance wavelengths, which can be individually addressed by crossed incident polarizations.
\end{abstract}

\section{INTRODUCTION}

In the last decades, properly designed photonic nanostructures have been used to control light at the subwavelength scale. ${ }^{1}$ With an appropriate choice of material, particle shape and size, it is possible to tailor many optical effects such as resonant scattering (e.g. for color rendering), ${ }^{2,3}$ directionality ${ }^{4,5}$ or polarization of scattered light, ${ }^{6}$ nano-scale heat generation ${ }^{7}$ or nonlinear optical effects. ${ }^{8-10}$ In this context, high-index dielectric nanostructures have attracted particular interest owing to their capacity to generate strong electric

Further author information: (Send correspondence to P.R.W.)

E-mail: peter.wiecha@cemes.fr and magnetic fields with very low non-radiative dissipative effects. ${ }^{11-15}$ Resonances in dielectric nanoparticles are spectrally tunable from the UV to the near IR. ${ }^{16,17}$

Conceiving photonic nanostructures is usually based on a reference geometry, chosen by intuitive considerations. The reference structure is then systematically adapted along a few free parameters in order to optimize its optical response to a given problem. If we consider more complex models, due to the increasing number of free parameters, the systematic analysis of the whole parameter-space can quickly become an impossible task and trial-and-error is usually not an efficient strategy neither. Heuristic techniques such as Evolutionary Optimization (EO) can be a better approach to this kind of problems. ${ }^{18}$ EO mimics natural selection processes in order to drive a solution to a complicated problem to its global optimum. While radio-frequency antenna design uses EO techniques already for several decades, in nano-photonics evolutionary methods are employed only for a couple of years. Some remarkable success has been reported in the design of single-particle geometries for the maximization of near-field intensities, ${ }^{19-22}$ in far-field scattering from plasmonic particles, ${ }^{23-25}$ for structural color ${ }^{26}$ or in the design of hybrid plasmonic/dielectric nanostructures. ${ }^{27}$ Evolutionary strategies have also been used to conceive larger structures like assemblies of several tens to hundreds of nano-particles, ${ }^{28}$ photonic crystal waveguides and metasurfaces ${ }^{29,30}$ or even complex nano-photonic devices. ${ }^{31,32}$ One major advantage of evolutionary optimization of nanostructure geometries is the possibility to include fabrication constraints in the problem description, hence automatically yielding experimentally feasible structures. ${ }^{33,34}$

The above cited works target single-objective problems. This is the simplest case of an optimization scenario in nano-optics. A nano-structure that concurrently fulfills multiple objectives will be in general more difficult to design. To this end, particular algorithms exist for evolutionary multi-objective optimization (EMO). ${ }^{35}$ Examples from recent literature are the automated design of multi-resonant silicon nanostructures, ${ }^{33}$ the optimization of a metasurface with multiple 
(a) the "direct" problem

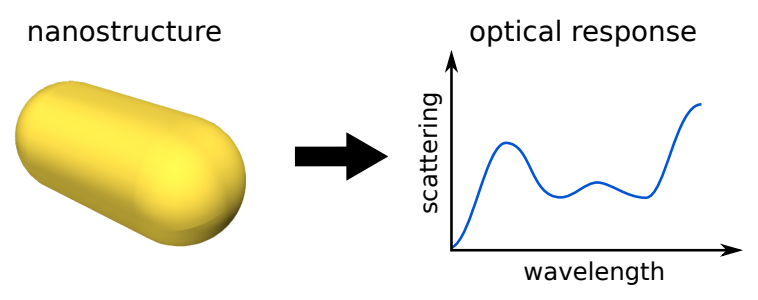

(b) the "inverse" problem

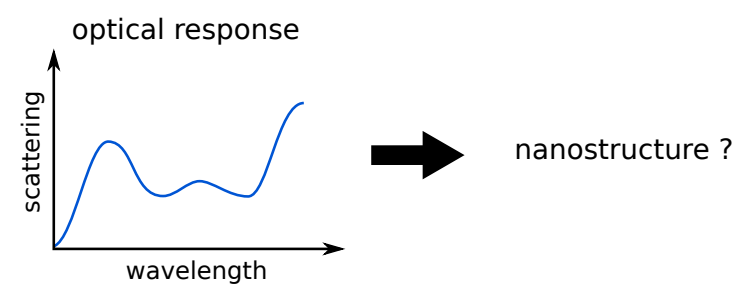

Figure 1. Illustration of both (a) direct and (b) inverse problems in nano-optics. The direct problem is the calculation of the optical response of a given nanostructure. The inverse problem, on the contrary, consists in searching a specific particle geometry which fulfills some desired optical response.

focal spots, ${ }^{36}$ or the EMO-driven design of a plasmonic waveguide which has an optimum robustness against geometrical variations. ${ }^{37}$

In this proceedings, we present a combination of EMO with the Green Dyadic Method (GDM) for fully retarded electro-dynamical simulations. ${ }^{38-40}$ We discuss two different approaches to multi-objective optimization. In the first, we use a multi-objective fitness function and optimize it using single-objective algorithms. In the second approach, we optimize all objectives concurrently via a Pareto optimization. We demonstrate both methods at the example of silicon nanoantennas, which are optimized to yield an optical response defined by two objectives.

\section{INVERSE PROBLEMS IN NANO-OPTICS}

Calculating the optical properties of photonic nanostructures with defined geometry and known material is a relatively straightforward problem. Manifold simulation techniques exist ranging from finite-difference time-domain (FDTD) to frequency domain techniques with surface or volume discretization. This is usually called the "direct problem" (see figure 1a).

Inverse problems (Fig. 1b), in which an optical response is defined in the first place and the nano-

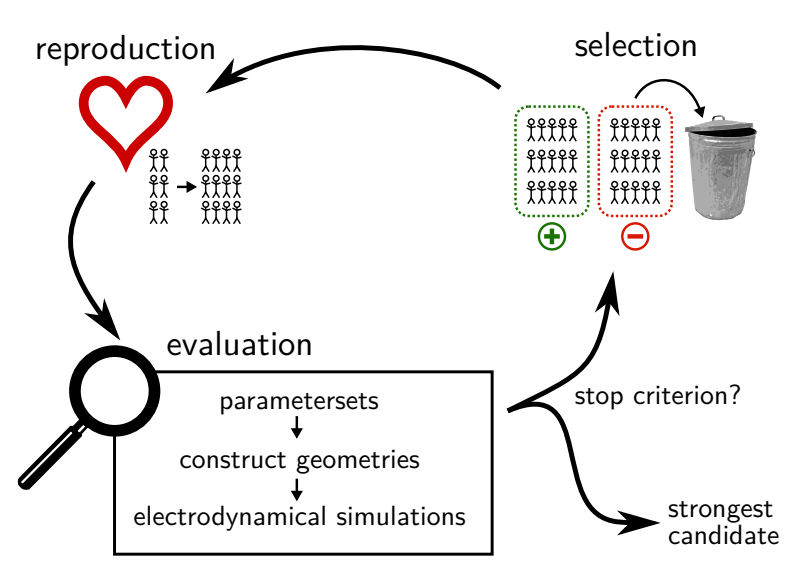

Figure 2. Sketch of the evolutionary optimization of photonic nanostructures. A population of parameter-sets, each describing the geometry of a photonic nanostructure undergoes a cycle of reproduction, evaluation and selection. In the illustration, each parameter-set is illustrated by a small matchstick man. Once a stop criterion (e.g. a maximum number of cycles) is met, the best candidate is chosen from the final set of solutions.

structure that yields such response is searched, are usually far more difficult to solve. To solve inverse problems in nano-optics, a reference geometry is usually chosen following intuitive reflections, which is subsequently studied systematically by varying only few free parameters. However, such approach often does not yield the very optimum solution and may even totally fail for complex problems for which the intuitive first step can no longer be used.

\section{EVOLUTIONARY OPTIMIZATION}

In such complex situations, a numerical optimization algorithm can help finding optimum solutions to very complicated problems. In the case of photonic nanostructure design, the objective function involves in general a numerical simulation of the optical response of the input nanostructure geometry. This leads to a non-analytic objective function which may not even be continuous within the limits of the parameter space. Hence, classical optimization algorithms fail, since they are based on the analytical evaluation of the objective function and its gradients.

A possible approach to find solutions to such complicated problems are heuristic algorithms like evolutionary optimization (EO). ${ }^{18}$ This class of algorithms mimics concepts of natural selection (survival of the fittest) and reproduction (mutation and cross-over of genes) to drive a population of parameters-sets for a problem to the optimum solution (see figure 2). 
Regular evolutionary optimization algorithms work on problems with a single optimization objective. Here, our goal is to design nanostructure geometries, such that several target properties are optimized concurrently. We therefore need a multi-objective optimization scheme to address the problem. The two most popular approaches are (1) the use of a single optimization function (often also called "fitness function"), combining several optimization objectives in a single value. ${ }^{18,36}$ Or (2) so-called Pareto optimization algorithms, which calculate a whole set of Pareto-optimal (or "non-dominated") solutions to the multi-objective problem. ${ }^{33,35,37}$

\subsection{Single-objective optimization: Multi-objective fitness function}

When using a single-objective optimization algorithm on multi-objective problems, there is one main difficulty. The necessary multi-objective fitness function is mostly not trivial to define. It must reflect all optimization goals in a finely tuned manner. If the different objective values are not well balanced within the multi-objective fitness function, the algorithm will end up running basically as a single-objective optimization on the most dominant target. However, for problems where the objectives can be easily balanced, the approach using a multi-objective fitness function is usually relatively simple to employ.

We demonstrate its principle on the design of a double-resonant silicon nanostructure. We search for a planar silicon structure consisting of up to four individual blocks. The ensemble shall have two different scattering resonances under illumination with an $X$, respectively $Y$ polarized plane wave. The free parameters are the positions, as well as the lengths and widths of the four blocks. The height is fixed to $100 \mathrm{~nm}$. Details on the structure model can be found in reference. ${ }^{33}$

We define a general shape of the desired scattering spectrum for both, $X$ and $Y$ polarization, as shown by thin lines with cross-shaped markers in figure 3 . In each evaluation step (see Fig. 2), the scattering cross-section is simulated using the GDM at each of the $N=10$ wavelengths of the predefined line-shape and for both polarizations. For the selection step (see Fig. 2), we calculate the $\chi^{2}$ value of each tested geometry:

$$
\chi^{2}=\sum_{i=1}^{N} \frac{\left(y_{\text {test }, i}-y_{\text {target }, i}\right)^{2}}{y_{\text {target }, i}},
$$

where the $y_{\text {target, } i}$ represent the target scattering intensities and $y_{\text {test }, i}$ are the normalized scattering crosssections of the simulated test geometries. The optimization goal is to find a structure which minimizes

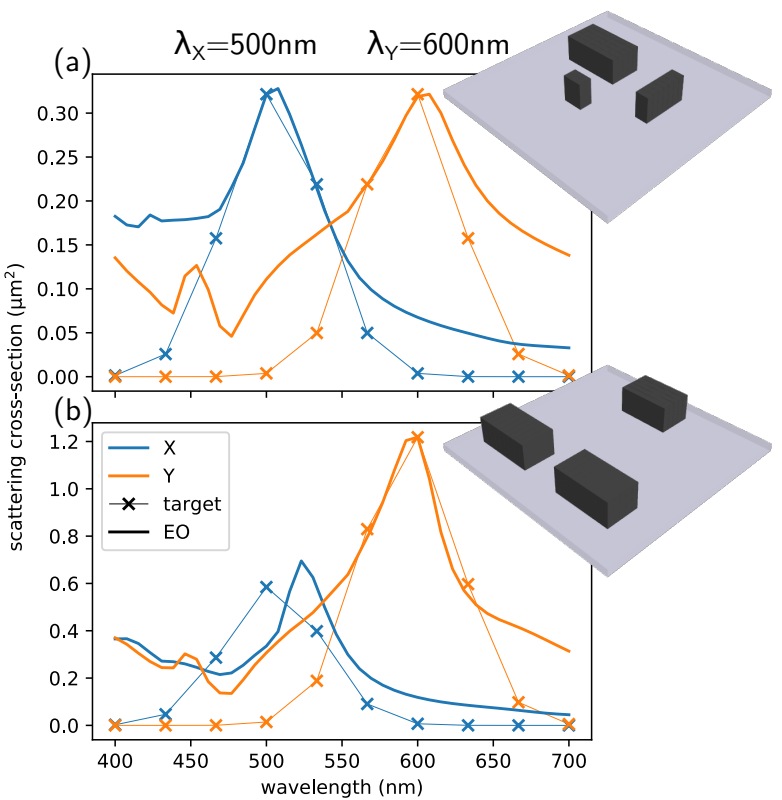

Figure 3. Result of the single-objective optimization using a two-objective fitness function. Optimization problems for (a) two target resonances with the same scattering intensity, and (b) two target resonances with a intensity ratio of $I_{X} / I_{Y}=0.5$. The structure illustrations show surfaces of $400 \times 400 \mathrm{~nm}^{2}$

equation (1). Hence, during the selection step, only structures with small values of $\chi^{2}$ are kept for the next iteration.

In figure 3 we show two optimization runs. In the first example, the two resonances are optimized to have equal amplitude. In the second example, we look for two resonances with relative intensity ratio of 0.5 . The optimization algorithm constructs geometries which approach the desired optical response. We point out that an advantage of this technique is the possibility to also tailor the line-width of a resonance. This might allow for instance to explore the range of achievable qualityfactors for certain geometric models of nano-resonators. We note furthermore, that we use the normalized spectra during the evaluation of equation (1). Our multiobjective fitness function therefore optimizes solely the shape of the scattering spectra, not their intensity. Due to the predefined line-shape which corresponds to a resonant profile, the optimization nevertheless yields resonant geometries.

We note, that in a similar approach multiple constraint functions can be used in a single-objective optimization. The different constraints are defined such that they effectively represent multiple objectives for 


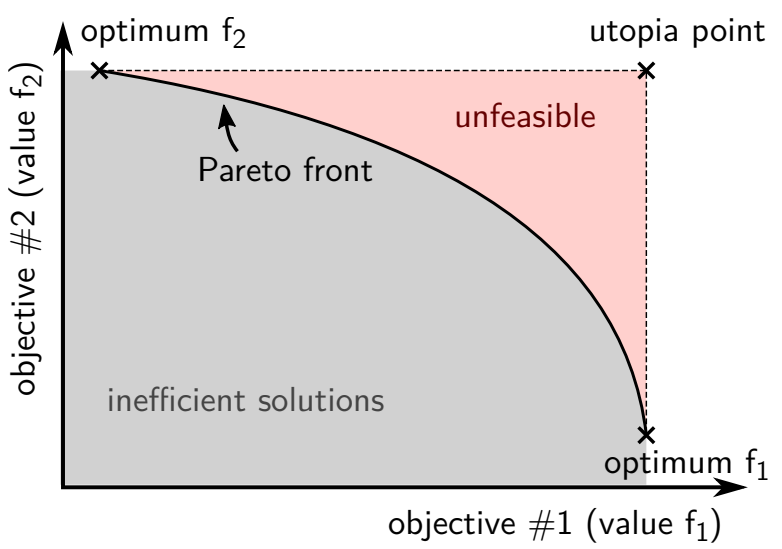

Figure 4. Illustration of the "Pareto front" concept. Several objectives are optimized simultaneously (2 objectives in this example). The "Pareto front" is composed by all solutions to the problem that cannot be further improved in one objective without worsening at least one other. A solution which combines the optimum individually reachable values for $f_{1}$ and $f_{2}$ can in reality not be achieved. It is hence called the "utopia point".

the optimization. ${ }^{32}$

\subsection{Multi-objective optimization: Pareto front}

A second approach to multi-objective problems is the so-called Pareto optimization. ${ }^{35}$ In this case, the objective functions are defined separately without need to bundle them to a single fitness value like the " $\chi$ " in the above example. For the Pareto optimization, specific evolutionary multi-objective optimization algorithms exist, which calculates the so-called "Pareto front". This Pareto front is the set of "non-dominated" solutions, in other words all solutions to the problem, that cannot be improved in all of the objectives without worsening at least one optimization objective (see figure 4). All non-dominated solutions on the Pareto front are in this manner optimally efficient.

To demonstrate the approach, we optimize again a planar silicon nanostructure geometry, using the same geometric model as in the previous section. We try to find a structure which concurrently has a scattering resonance at $\lambda_{X}=450 \mathrm{~nm}$ for $X$-polarized plane wave illumination and a resonance at $\lambda_{Y}=570 \mathrm{~nm}$ for $Y$ polarization. The optimization target in this second example shall be the scattering efficiency, which is defined as the scattering cross-section divided by the geometrical cross-section of the structure (its "footprint"):

$$
Q_{\text {scat }}=\frac{\sigma_{\text {scat }}}{\sigma_{\text {geo }}} .
$$
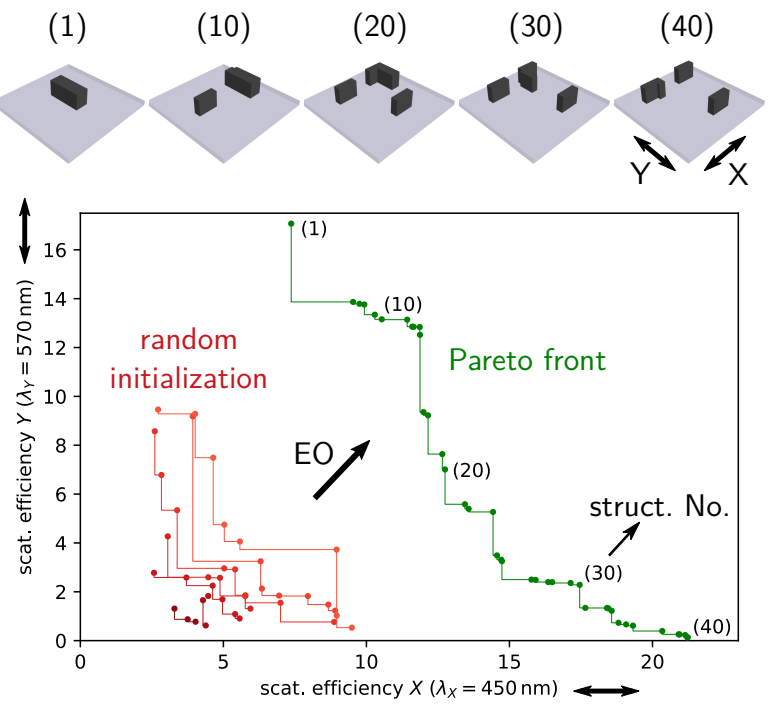

Figure 5. Initial random population (red points) and Pareto front (green points) after a multi-objective optimization using $\lambda_{X}=450 \mathrm{~nm}$ and $\lambda_{Y}=570 \mathrm{~nm}$. Selected structure geometries from the Pareto front are illustrated at the top $\left(300 \times 300 \mathrm{~nm}^{2}\right)$.

Technical details on the optimization algorithms and model can be found in reference, ${ }^{33}$ in which we have described the design of similar nanostructures for different resonant wavelengths. We note that in contrast to the former section, the Pareto optimization yields a true maximization of the scattering intensity at the specific wavelengths.

The Pareto front at the end of the optimization is shown in figure 5 (green line and points). In the same figure, the fitness values of both objective functions of the random initial population are shown as red points. Geometries of selected structures on the Pareto front are shown at the top of the figure. The EMO was able to find structures that significantly increase the scattering for both target resonances. We see that the general alignment of the structures follows the polarization, defined for the target resonance. For the shorter wavelength $\left(\lambda_{X}=450 \mathrm{~nm}\right)$, the optimum structure furthermore consists of several blocks, while the longer wavelength yields one large structure, unifying all available blocks in a single element. This is a result of the constrained size of the four constituents and is discussed in detail in reference. ${ }^{33}$ Finally, in figure 6 we show the spectra of all structures on the Pareto front. Indeed, the first structure (bottom of figure 6, number "1", dark blue line) has a pronounced optical resonance at $\lambda=570 \mathrm{~nm}$ under $Y$ polarization, while the scattering at the complementary objective $(\lambda=450 \mathrm{~nm}$, 


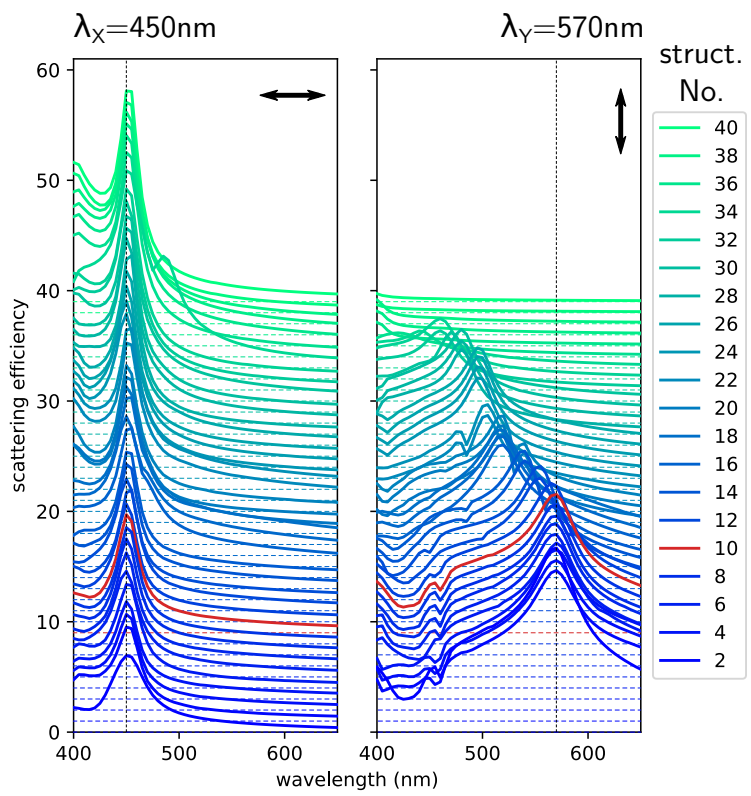

Figure 6. Spectra of all structures on the Pareto front after the multi-objective optimization with $\lambda_{X}=450 \mathrm{~nm}$ and $\lambda_{Y}=570 \mathrm{~nm}$ (see also figure 5). The scattering efficiency is shown for $X$ polarized plane wave illumination on the left plot and for $Y$ polarization on the right. The best compromise structure is the number "10", highlighted by red lines. Each successive spectrum is offset on the $Y$-scale by a positive step of 1 . Hence, for example to get the actual scattering efficiency of structure " 20 ", 19 must be subtracted from the shown values.

$X$-polarization) is weak. The structure at the other end of the Pareto front yields the opposite image: a very strong resonance at $\lambda=450 \mathrm{~nm}$ occurs for $X$ polarization, while $Y$-polarized light is barely scattered (top, purple line in figure 6). In the "middle" of the Pareto-front, structures are found which yield resonances in both cases and can be considered a compromise between the two objective functions.

\section{CONCLUSION}

In conclusion, we demonstrated two different approaches to multi-objective optimization of photonic nanostructures. The first approach is based on single-objective optimization algorithms, using a multiobjective fitness function, which summarized multiple optimization objectives in a single number. This method can be used to design the line-shape of a spectral response over a larger wavelength range, which effectively treats a problem of a large number of objectives ( $N$ considered wavelengths). However, such approach becomes limited when several entirely different quantities are used as optimization objectives, since the balancing of the different target values is crucial for the multi-objective fitness function. Hence, we presented a second approach to multi-objective, namely Pareto optimization, which can be a promising alternative in cases of very different target functions. Pareto optimization consists in the search of the set of nondominated solutions to the problem. These solutions cannot be further optimized in all objective functions simultaneously. We demonstrated both approaches at the example of a multi-resonant silicon nanostructure, yielding comparable results.

In the future, EMO could be applied on many problems in nano-optics, which would profit from efficient schemes to solve inverse problems. Examples go far beyond multiresonant antennas ${ }^{41}$ or polarization dependent tailored optical behavior. ${ }^{42,43}$ For instance nanoantennas for optimized nonlinear optical effects are particularly promising with respect to inverse design methods. For the maximization of optical second harmonic generation for instance, ${ }^{44,45}$ strong resonances could be concurrently designed at the fundamental and harmonic frequencies and furthermore tailored to yield strongest field-enhancements just at the surface of the nanoparticle.

\section{ACKNOWLEDGMENTS}

This work was supported by Programme Investissements d'Avenir under the program ANR-11-IDEX0002-02, reference ANR-10-LABX-0037-NEXT, by the LAAS-CNRS micro and nanotechnologies platform, a member of the French RENATECH network and by the computing facility center CALMIP of the University of Toulouse under grant P12167.

\section{REFERENCES}

[1] Novotny, L. and Hecht, B., [Principles of NanoOptics], Cambridge University Press, Cambridge ; New York (2006).

[2] Tan, S. J., Zhang, L., Zhu, D., Goh, X. M., Wang, Y. M., Kumar, K., Qiu, C.-W., and Yang, J. K. W., "Plasmonic Color Palettes for Photorealistic Printing with Aluminum Nanostructures," Nano Letters 14, 4023-4029 (July 2014).

[3] Duempelmann, L., Casari, D., Luu-Dinh, A., Gallinet, B., and Novotny, L., "Color Rendering Plasmonic Aluminum Substrates with Angular Symmetry Breaking," ACS Nano 9, 12383-12391 (Dec. 2015). 
[4] Albella, P., Shibanuma, T., and Maier, S. A., "Switchable directional scattering of electromagnetic radiation with subwavelength asymmetric silicon dimers," Scientific Reports 5, 18322 (Dec. 2015).

[5] Lindfors, K., Dregely, D., Lippitz, M., Engheta, N., Totzeck, M., and Giessen, H., "Imaging and Steering Unidirectional Emission from Nanoantenna Array Metasurfaces," ACS Photonics 3, 286-292 (Feb. 2016).

[6] Wiecha, P. R., Black, L.-J., Wang, Y., Paillard, V., Girard, C., Muskens, O. L., and Arbouet, A., "Polarization conversion in plasmonic nanoantennas for metasurfaces using structural asymmetry and mode hybridization," Scientific Reports 7, 40906 (Jan. 2017).

[7] Baffou, G., Quidant, R., and Girard, C., "Heat generation in plasmonic nanostructures: Influence of morphology," Applied Physics Letters 94, 153109 (Apr. 2009).

[8] Kauranen, M. and Zayats, A. V., "Nonlinear plasmonics," Nature Photonics 6, 737-748 (Nov. 2012).

[9] Black, L.-J., Wiecha, P. R., Wang, Y., de Groot, C. H., Paillard, V., Girard, C., Muskens, O. L., and Arbouet, A., "Tailoring Second-Harmonic Generation in Single L-Shaped Plasmonic Nanoantennas from the Capacitive to Conductive Coupling Regime," ACS Photonics 2, 1592-1601 (Nov. 2015).

[10] Wiecha, P. R., Arbouet, A., Kallel, H., Periwal, P., Baron, T., and Paillard, V., "Enhanced nonlinear optical response from individual silicon nanowires," Physical Review B 91, 121416 (Mar. 2015).

[11] Ginn, J. C., Brener, I., Peters, D. W., Wendt, J. R., Stevens, J. O., Hines, P. F., Basilio, L. I., Warne, L. K., Ihlefeld, J. F., Clem, P. G., and Sinclair, M. B., "Realizing Optical Magnetism from Dielectric Metamaterials," Physical Review Letters 108, 097402 (Feb. 2012).

[12] Albella, P., Poyli, M. A., Schmidt, M. K., Maier, S. A., Moreno, F., Sáenz, J. J., and Aizpurua, J., "Low-Loss Electric and Magnetic Field-Enhanced Spectroscopy with Subwavelength Silicon Dimers," The Journal of Physical Chemistry C 117, 1357313584 (July 2013).

[13] Albella, P., Alcaraz de la Osa, R., Moreno, F., and Maier, S. A., "Electric and Magnetic Field Enhancement with Ultralow Heat Radiation Dielectric Nanoantennas: Considerations for SurfaceEnhanced Spectroscopies," ACS Photonics 1, 524529 (June 2014).
[14] Bakker, R. M., Permyakov, D., Yu, Y. F., Markovich, D., Paniagua-Domínguez, R., Gonzaga, L., Samusev, A., Kivshar, Y., Luk'yanchuk, B., and Kuznetsov, A. I., "Magnetic and Electric Hotspots with Silicon Nanodimers," Nano Letters 15, 2137-2142 (Mar. 2015).

[15] Kuznetsov, A. I., Miroshnichenko, A. E., Brongersma, M. L., Kivshar, Y. S., and Luk'yanchuk, B., "Optically resonant dielectric nanostructures," Science 354 (Nov. 2016).

[16] Cao, L., Fan, P., Barnard, E. S., Brown, A. M., and Brongersma, M. L., "Tuning the Color of Silicon Nanostructures," Nano Letters 10, 2649-2654 (July 2010).

[17] Zhao, W., Liu, B., Jiang, H., Song, J., Pei, Y., and Jiang, Y., "Full-color hologram using spatial multiplexing of dielectric metasurface," Optics Letters 41, 147 (Jan. 2016).

[18] Sivanandam, S. and Deepa, S., [Introduction to Genetic Algorithms], Springer, Heidelberg (2008).

[19] Forestiere, C., Donelli, M., Walsh, G. F., Zeni, E., Miano, G., and Dal Negro, L., "Particle-swarm optimization of broadband nanoplasmonic arrays," Optics Letters 35, 133 (Jan. 2010).

[20] Feichtner, T., Selig, O., Kiunke, M., and Hecht, B., "Evolutionary Optimization of Optical Antennas," Physical Review Letters 109, 127701 (Sept. 2012).

[21] Forestiere, C., Pasquale, A. J., Capretti, A., Miano, G., Tamburrino, A., Lee, S. Y., Reinhard, B. M., and Dal Negro, L., "Genetically Engineered Plasmonic Nanoarrays," Nano Letters 12, 20372044 (Apr. 2012).

[22] Forestiere, C., He, Y., Wang, R., Kirby, R. M., and Dal Negro, L., "Inverse Design of Metal Nanoparticles' Morphology," ACS Photonics 3, 68-78 (Jan. 2016)

[23] Ginzburg, P., Berkovitch, N., Nevet, A., Shor, I., and Orenstein, M., "Resonances On-Demand for Plasmonic Nano-Particles," Nano Letters 11, 2329-2333 (June 2011).

[24] Tassadit, A., Macías, D., Sánchez-Gil, J. A., Adam, P. M., and Rodriguez-Oliveros, R., "Metal nanostars: Stochastic optimization of resonant scattering properties," Superlattices and Microstructures 49, 288-293 (Mar. 2011).

[25] Macías, D., Adam, P.-M., Ruíz-Cortés, V., Rodríguez-Oliveros, R., and Sánchez-Gil, J. A., "Heuristic optimization for the design of plasmonic nanowires with specific resonant and scattering properties," Optics Express 20, 13146 (June 2012). 
[26] Andkjær, J., Johansen, V. E., Friis, K. S., and Sigmund, O., "Inverse design of nanostructured surfaces for color effects," JOSA B 31, 164-174 (Jan. 2014).

[27] Bigourdan, F., Marquier, F., Hugonin, J.-P., and Greffet, J.-J., "Design of highly efficient metallodielectric patch antennas for single-photon emission," Optics Express 22, 2337 (Feb. 2014).

[28] Wiecha, P. R., Arbouet, A., Cuche, A., Paillard, V., and Girard, C., "Decay rate of magnetic dipoles near nonmagnetic nanostructures," Physical Review B 97, 085411 (Feb. 2018).

[29] Jensen, J. and Sigmund, O., "Topology optimization for nano-photonics," Laser \& Photonics Reviews 5, 308-321 (Mar. 2011).

[30] Girard, C., Wiecha, P. R., Cuche, A., and Dujardin, E., "Designing Thermoplasmonic Properties of Metallic Metasurfaces," arXiv:1804.01111 [cond-mat, physics:physics] (Apr. 2018).

[31] Lu, J. and Vučković, J., "Nanophotonic computational design," Optics Express 21, 13351-13367 (June 2013).

[32] Piggott, A. Y., Lu, J., Lagoudakis, K. G., Petykiewicz, J., Babinec, T. M., and Vučković, J., "Inverse design and demonstration of a compact and broadband on-chip wavelength demultiplexer," Nature Photonics 9, 374-377 (June 2015).

[33] Wiecha, P. R., Arbouet, A., Girard, C., Lecestre, A., Larrieu, G., and Paillard, V., "Evolutionary multi-objective optimization of colour pixels based on dielectric nanoantennas," Nature Nanotechnology 12, 163-169 (Feb. 2017).

[34] Feichtner, T., Selig, O., and Hecht, B., "Plasmonic nanoantenna design and fabrication based on evolutionary optimization," Optics Express 25, 10828-10842 (May 2017).

[35] Deb, K., [Multi-Objective Optimization Using Evolutionary Algorithms], vol. 16, Wiley (2001).

[36] Hu, J., Ren, X., Reed, A. N., Reese, T., Rhee, D., Howe, B., Lauhon, L. J., Urbas, A. M., and Odom, T. W., "Evolutionary Design and Prototyping of Single Crystalline Titanium Nitride Lattice Optics," ACS Photonics 4, 606-612 (Mar. 2017).

[37] Jung, J., "Robust Design of Plasmonic Waveguide Using Gradient Index and Multiobjective Optimization," IEEE Photonics Technology Letters 28, 756-758 (Apr. 2016).

[38] Girard, C., "Near fields in nanostructures," Reports on Progress in Physics 68, 1883-1933 (Aug. 2005).
[39] Martin, O. J. F., Girard, C., and Dereux, A., "Generalized Field Propagator for Electromagnetic Scattering and Light Confinement," Physical Review Letters 74, 526-529 (Jan. 1995).

[40] Wiecha, P. R., "pyGDM - A python toolkit for full-field electro-dynamical simulations and evolutionary optimization of nanostructures," arXiv:1802.04071 [cond-mat, physics:physics] (Feb. 2018).

[41] Aouani, H., Šípová, H., Rahmani, M., NavarroCia, M., Hegnerová, K., Homola, J., Hong, M., and Maier, S. A., "Ultrasensitive Broadband Probing of Molecular Vibrational Modes with Multifrequency Optical Antennas," ACS Nano 7, 669-675 (Jan. 2013).

[42] Gao, Z. and Wang, Z.-Y., "Terahertz Plasmonic Cross Resonant Antenna," Journal of Electromagnetic Waves and Applications 25, 1730-1739 (Jan. 2011).

[43] Dopf, K., Moosmann, C., Kettlitz, S. W., Schwab, P. M., Ilin, K., Siegel, M., Lemmer, U., and Eisler, H.-J., "Coupled T-Shaped Optical Antennas with Two Resonances Localized in a Common Nanogap," ACS Photonics 2, 1644-1651 (Nov. 2015)

[44] Harutyunyan, H., Volpe, G., Quidant, R., and Novotny, L., "Enhancing the Nonlinear Optical Response Using Multifrequency Gold-Nanowire Antennas," Physical Review Letters 108, 217403 (May 2012).

[45] Celebrano, M., Wu, X., Baselli, M., Großmann, S., Biagioni, P., Locatelli, A., De Angelis, C., Cerullo, G., Osellame, R., Hecht, B., Duò, L., Ciccacci, F., and Finazzi, M., "Mode matching in multiresonant plasmonic nanoantennas for enhanced second harmonic generation," Nature Nanotechnology 10, 412-417 (May 2015). 